\title{
Effect of Continuous Positive Airway Pressure on Chronic Cough in Patients with Obstructive Sleep Apnea and Concomitant Gastroesophageal Reflux
}

\author{
Jiao Su $\mathbb{D}^{\prime}$ \\ Yifei Fang' \\ Yang Meng' \\ Chunling Zhao' \\ Yanjun Liu' \\ Linge Sun \\ Mengge Wang' \\ Liping Dai ${ }^{2}$ \\ Songyun Ouyang' \\ 'Department of Respiratory and Sleep \\ Medicine, First Affiliated Hospital of \\ Zhengzhou University, Zhengzhou, \\ Henan, 450052, People's Republic of \\ China; ${ }^{2}$ Henan Institute of Medical and \\ Pharmaceutical Sciences, Zhengzhou \\ University, Zhengzhou, Henan, 450052, \\ People's Republic of China
}

Correspondence: Songyun Ouyang Department of Respiratory and Sleep Medicine, First Affiliated Hospital of Zhengzhou University, Zhengzhou, Henan, 450052, People's Republic of China

Tel +8637l-6629 509l

Email ouyangsy@163.com
Background: Obstructive sleep apnea (OSA) and/or gastroesophageal reflux (GER) may be the contributors to chronic cough in patients with OSA and concomitant GER. This study aimed to explore whether continuous positive airway pressure (CPAP), antireflux treatment and lifestyle modifications improve chronic cough in patients with OSA and concomitant GER.

Methods: Patients with OSA and concomitant GER who also experienced chronic cough were enrolled, and were divided into two groups. Patients who were treated with general treatment (antireflux treatment and lifestyle modifications) as the control group, and patients who were treated with CPAP and general treatment as the treatment group. Effects of different treatments on chronic cough were assessed, and the association among chronic cough, GER and OSA was evaluated by Pearson's correlation analysis.

Results: The Pittsburgh Sleep Quality Index and Epworth Sleepiness Scale were better in the treatment group after 1 week of treatment; further, the improvement became stronger with the duration of therapy. The gastroesophageal reflux disease questionnaire, visual analog scale (VAS) for cough, and daytime and nighttime cough symptom scores significantly improved in both groups after treatment, whereas this improvement was more significant in the treatment group. Significant associations between the apnea-hypopnea index (AHI) and VAS, weak acid reflux and VAS, and weak acid reflux and AHI were observed.

Conclusion: CPAP improved the symptoms of chronic cough and GER in patients with OSA and concomitant GER. AHI and weak acid reflux may be important factors affecting the therapeutic effect of chronic cough in patients with OSA and concomitant GER.

Keywords: obstructive sleep apnea, gastroesophageal reflux, chronic cough, continuous positive airway pressure

\section{Introduction}

Chronic cough affects $2.3-33 \%$ of adults ${ }^{1,2}$ and is a common complaint in respiratory outpatient clinic visits. Obstructive sleep apnea (OSA) could cause a variety of complications, such as chronic cough, gastroesophageal reflux (GER), cardiovascular and cerebrovascular diseases. ${ }^{3-6}$ A cross-sectional study found that the incidence of chronic cough was up to $33 \%$ in patients with sleep-disordered breathing. ${ }^{7}$ Shi et al have found that OSA can induce cough hypersensitivity. ${ }^{8}$ OSA is increasingly thought to be the cause of chronic cough. ${ }^{3}$ However, the pathogenesis of chronic cough in patients with OSA is multifactorial.

The incidence of GER is high in patients with OSA, 9,10 and GER may be a contributor to chronic cough in patients with OSA and GER. A multivariate 
population-level analysis suggested that there was a significant correlation between GER and OSA after the exclusion of other confounders. ${ }^{11}$ The CHEST Guideline and Expert Panel Report published in Chest has suggested that general treatment, including lifestyle modifications and weight reduction, alleviates the symptom of chronic cough in patients with GER. ${ }^{12}$ Tamanna et al have found that continuous positive airway pressure (CPAP) can improve the events of nocturnal GER in OSA patients. ${ }^{13}$ A retrospective study has demonstrated that GER is present in $42 \%$ of patients with OSA, and CPAP can significantly improve GER events. ${ }^{14}$ A Randomized Controlled Pilot Study found that CPAP treatment could relieve the symptom of chronic cough in OSA patients. ${ }^{15}$

Unfortunately, as far as OSA and GER-related cough are concerned, the therapeutic management of chronic cough remains largely empirical. This study aimed to explore effects of different treatments on chronic cough in patients with OSA and concomitant GER and the possible mechanism of this association.

\section{Methods}

\section{Participants}

This retrospective study enrolled patients aged $\geq 18$ years with chronic cough who visited the Respiratory and Sleep Medicine Department of First Affiliated Hospital of Zhengzhou University between March 2017 and November 2020. Informed consent was waived owing to the retrospective nature of the study. The patient data accessed was maintained with confidentiality. This study was conducted in accordance with the World Medical Association Declaration of Helsinki and approved by the grass-roots ethics committee of the First Affiliated Hospital of Zhengzhou University.

The inclusion criteria were: (1) cough more than 8 weeks; (2) normal spirometry and provocation test; (3) normal chest radiographs and/or computed tomography scans of the chest; (4) normal acidophilic granulocyte count of induced sputum; (5) normal skin prick test; (6) Tests for the diagnosis of upper airway disease were normal; ${ }^{16}$ (7) no history of angiotensin-converting enzyme inhibitor drug use.

The exclusion criteria were: (1) the unstable haemodynamics patients, acute stage of cardiovascular and cerebrovascular diseases, uncontrollable arrhythmia, congestive heart failure; (2) giant bullous emphysema; (3) a history of hypertension, poor blood pressure control;
(4) esophagus cancer, or erosive esophagitis; (5) heavy smoker; ${ }^{17}$ (6) alcohol dependence and alcohol abuse; ${ }^{18}$ (7) pregnancy.

\section{Pittsburgh Sleep Quality Index}

The Pittsburgh Sleep Quality Index (PSQI) was used to assess the subjective sleep quality of the participants within the past 1 month. ${ }^{19}$ It consists of 19 selfassessment items and 5 other-assessment items, in which 18 self-assessment items were scored. The 18 selfassessment items were divided into 7 aspects, and each answer is graded on a scale of 0 to 3 . The total score ranges from 0 to 21 , with higher score indicating poorer sleep quality.

\section{Epworth Sleepiness Scale}

The Epworth Sleepiness Scale (ESS) score was used to assess daytime sleepiness. ${ }^{20}$ It consists of 8 questions regarding how often an individual dozes during daily activities. In this study, a Chinese version of the ESS score was used to assess daytime sleepiness of patients in both groups. Each answer is scored on a scale of 0 to 3 . The ESS score ranges from 0 (never dozed) to 24 (high chance of dozing), with higher values indicating greater sleep propensity during the day.

\section{Gastroesophageal Reflux Disease Questionnaire}

The gastroesophageal reflux disease questionnaire (GERD-Q) can be used to assess the severity of reflux symptoms. ${ }^{21}$ It consists of six questions that are divided into the following three aspects: heartburn, reflux, epigastric pain, nausea, sleep disorder and antireflux treatment use within the past 1 week. In this study, a Chinese version of the GERD-Q was used to assess GER symptoms of patients in both groups. A total score of $\geq 8$ was considered indicative of GER disease.

\section{Visual Analog Scale for Cough}

The visual analog scale (VAS) for cough ${ }^{16}$ is used to assess cough severity by the linear integral method. It ranges from 0 to 100 , with 0 indicating asymptomatic and 100 indicating the most severe cough. It can be used for the longitudinal comparison of cough severity before and after the treatment. 


\section{Cough Symptom Score}

The Chinese version of the cough symptom score was used to evaluate daytime cough symptoms and nighttime cough symptoms separately according to the 2015 Chinese Cough Diagnosis and Treatment Guidelines. ${ }^{16}$ Each score is rated on a scale of 0 (no cough) to 3 (most severe cough), with higher values indicating more severe cough symptoms.

\section{4-h Esophageal Multichannel Intraluminal Impedance-pH}

24-h esophageal multichannel intraluminal impedance-pH (24-h MII-pH) (Medtronic Inc, USA) monitoring was used to diagnose GER. No antacid treatment was administered during the examination and 8 weeks prior to the examination. An abnormal GER was defined by a DeMeester score of $\geq 14.72^{22}$ and/or meeting the criteria of Lyon Consensus. ${ }^{23}$ Acid reflux was defined as nadir $\mathrm{pH}<4$, weak acid reflux was define as nadir $\mathrm{pH}$ between 4 and 7 , and non-acid reflux was define as nadir $\mathrm{pH}>7 .{ }^{24}$

\section{Polysomnography}

Overnight polysomnography monitoring was performed using a Compumedics system (E-series compumedics, Australia). Sleep stages and respiratory events were classified according to the American Academy of Sleep Medicine standards (version 2.4). ${ }^{25}$ The apnea-hypopnea index (AHI) was used to define the severity of OSA.

$24 \mathrm{~h}$ MII-pH combined with polysomnography monitoring were performed for each patient at the baseline. Synchronous $\mathrm{pH}$ signals were recorded in the reflux monitoring and Compumedics systems to accurately analyze the temporal association between apnea and reflux events.

\section{Lifestyle Modifications}

Lifestyle modifications included diet control, aerobic exercises, tongue exercises, soft palate exercises, exercises for the cheeks, throat, and neck, exercises for lips and jaw, sleeping on one's side, and quit smoking and alcohol.

\section{Diet Control}

Diet control referred to calorie intake and dietary pattern changes. $^{26,27}$ Low-carbohydrate diets come in several forms, including moderate to very low-carbohydrate diets. The moderate carbohydrate intake provided about $26 \sim 5 \%$ of total calories. The term "low-fat diet" mainly referred to a diet low in triglycerides and cholesterol. This study considered the moderate carbohydrate and low-fat diet patterns, with daily caloric intake ranging from 1200 to $1500 \mathrm{kcal}$ for women and 1500 to $1800 \mathrm{kcal}$ for men.

\section{Aerobic Exercises}

Aerobic exercises ${ }^{28,29}$ included walking, jogging, gymnastics, and treadmill exercise. The aerobic exercises intensity was recommended to be $60 \% \sim 85 \%$ of heart rate reserve, 4 times per week, 150 min per time, and the exercise intensity was adjusted based on the patients' cardiopulmonary exercise test results.

\section{Tongue Exercises}

1. Keep the chin open throughout the exercise. Press the front of the tongue against the hard palate for 5 $\mathrm{s}$, then relax for $8 \mathrm{~s}, 10$ times a cycle, 3 cycles daily.

2. Open the mouth and try to touch the chin with the tip of the tongue. Hold for $5 \mathrm{~s}$, relax for $8 \mathrm{~s}, 10$ times a cycle, 3 times daily.

3. Open the mouth, try to touch the tip of the nose with the tip of the tongue, and relax for $8 \mathrm{~s}, 10$ times a cycle, 3 cycles daily. ${ }^{30}$

\section{Soft Palate Exercises}

1. Pronounce "A, E, I, O, U" 5 times daily.

2. Inhale through the nose and exhale through the mouth, keeping the lips together while exhaling for $5 \mathrm{~s}$. Repeat 5 times, 3 times daily. ${ }^{30}$

\section{Cheeks, Throat, and Neck Exercises}

1. Tilt the head back and stick out the tongue as far as possible with the tip of the tongue. Hold for $5 \mathrm{~s}$, return the head to the starting position, and relax the tongue for 8s. Repeat the process 5 times daily.

2. Tilt the head back and gently bite the tongue. Attempt swallowing once. Then, stand the head upright and relax the tongue for 8s. Repeat the process 5 times daily.

3. Facial muscle training used the facial expression simulation method to exercise orbicularis oris muscle, buccinator muscle, as well as lateral and medial pterygoid muscles. ${ }^{30}$

\section{Lips and Jaw Exercises}

1. Shrink lips for $10 \mathrm{~s}$, then relax for $12 \mathrm{~s}$ repeat 10 times once daily.

2. Open mouth wide while shrinking the lips. Hold for $5 \mathrm{~s}$ and then relax for $8 \mathrm{~s}$. Repeat this 5 times daily.

3. Place one hand under the chin, then open the mouth as wide as possible. Hold for $5 \mathrm{~s}$, then relax for 8 s. Repeat this 10 times a day. ${ }^{30}$ 


\section{Smoking}

The World Health Organization ${ }^{17}$ define smoking as continuous or cumulative smoking for 6 months or more. Smokers were further classified as regular smoker (smoking more than one cigarette daily for consecutive or cumulative six months), occasional smoker (smoking more than four times per week, but less than one cigarette per day), and non-smoker. Passive smoking is defined as inhaling smoke from a smoker at least $15 \mathrm{~min} /$ day for more than one day per week.

\section{Protocol}

According to the above inclusion and exclusion criteria, patients with chronic cough who met the criteria were enrolled. After relevant auxiliary examinations, patients with OSA and concomitant GER were further collected. General treatment included antireflux treatment (proton pump inhibitor \pm mosapride) and lifestyle modifications. Average CPAP use $\geq 4 \mathrm{~h}$ per night was considered as good compliance, average CPAP use $<2 \mathrm{~h}$ per night was considered as poor compliance. ${ }^{31,32}$ Patients with poor CPAP compliance but received general treatment as the control group, patients with good CPAP compliance and received general treatment as the treatment group. Patients kept a daily treatment log and, under the supervision of physicians, through regular outpatient visits to ensure the completion of treatment. The study protocol is presented in Figure 1.

\section{Statistical Analysis}

Data are presented as mean \pm SD. First, a Chi-square test was used to assess whether the variables of sex, smoking, and alcohol consumption had differences between the treatment and control groups. Second, as the overnight polysomnography and 24-h MII-pH monitoring were performed only before the treatment, an independent sample $t$-test was used to analyze AHI, the longest time of apnea, the lowest oxygen saturation, DeMeester score, reflux measures between the treatment and control groups. It could be used to assess whether there were significant differences in relevant indicators between the treatment and control groups, and to ensure the homogeneity of the patients of the two groups before treatment. Third, a analysis of variance of repeated measurement data were used to assess of the improvement of PSQI, ESS, GERD-Q, VAS and cough symptom scores. Forth, because of the collinearity between independent variables which

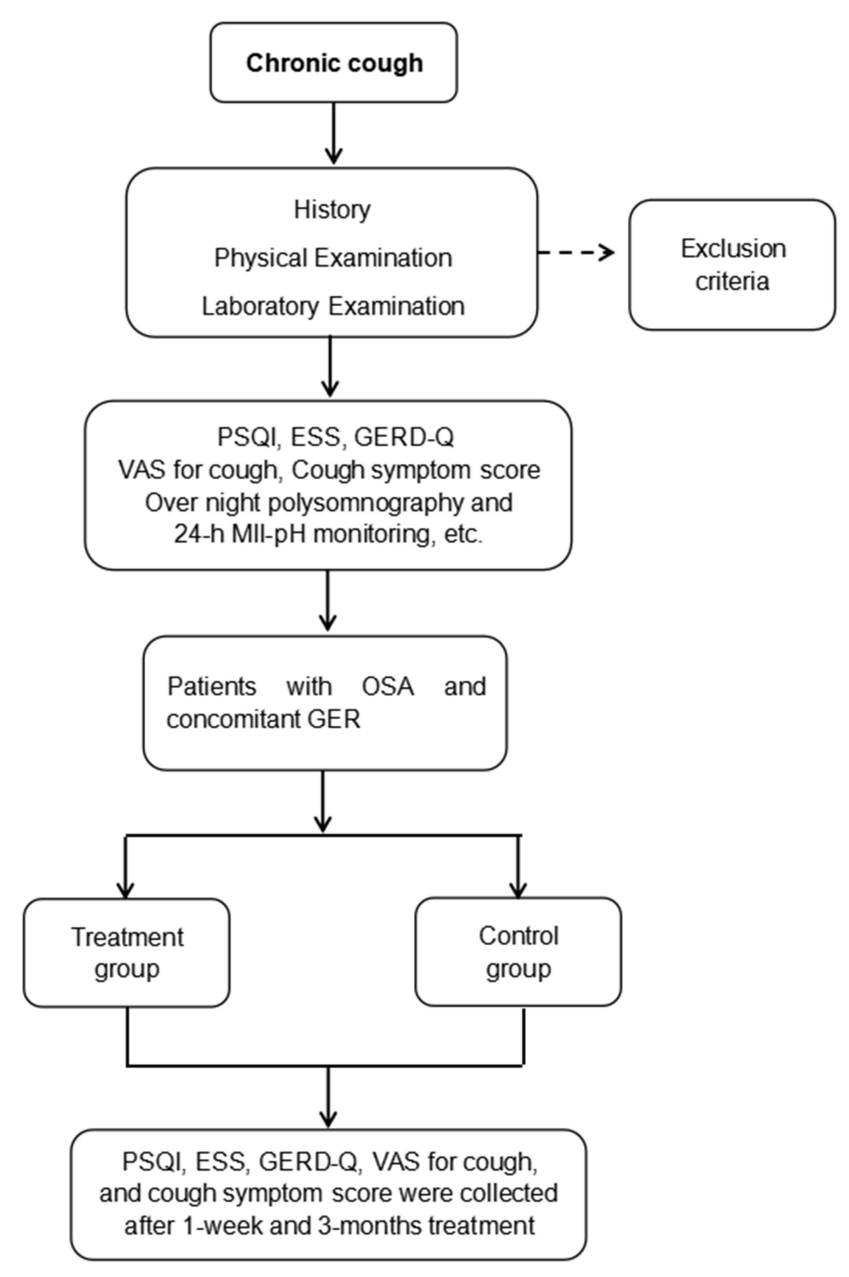

Figure I The protocol of the study.

entered in the model, the regression model was not developed. Pearson's correlation analysis was used to explore the association between VAS for cough and age, BMI, PSQI, ESS, GERD-Q, objective sleep and reflux-related indicators. $P<0.05$ was considered statistically significant. All statistical analyses were performed using the Statistics Package for Social Science version 16.0 (SPSS Inc., Chicago, IL, USA).

\section{Results}

\section{Baseline Characteristics}

Of the 86 patients who met the criteria, 40 patients in the control group and 46 patients in the treatment group. The pre-treatment data of this study are presented in Table 1. Patients in both groups had poor sleep quality, severe daytime sleepiness, sleep structural disorder, decreased sleep efficiency, moderate-severe sleep apnea, significant weak acid reflux, and moderate coughing events. These 
Table I The Baseline Data of This Study

\begin{tabular}{|c|c|c|c|}
\hline & Treatment Group $(n=46)$ & Control Group $(n=40)$ & $P$ value* \\
\hline Age & $36.91 \pm 5.55$ & $37.52 \pm 5.92$ & $0.62^{\mathrm{a}}$ \\
\hline Sex (Male/Female) & $26 / 20$ & $24 / 16$ & $0.74^{\mathrm{b}}$ \\
\hline BMI $\left(\mathrm{kg} / \mathrm{m}^{2}\right)$ & $27.48 \pm 4.75$ & $28.38 \pm 4.94$ & $0.16^{\mathrm{a}}$ \\
\hline Smoking: & & & $0.72^{\mathrm{b}}$ \\
\hline Yes & $26(56.52 \%)$ & $25(62.50 \%)$ & \\
\hline Regular smoker & 0 & 0 & \\
\hline Occasional smoker & $19(41.30 \%)$ & $20(50.00 \%)$ & \\
\hline Passive smoking & $7(15.22 \%)$ & $5(12.50 \%)$ & \\
\hline Non-smoker & $20(43.48 \%)$ & $15(37.50 \%)$ & \\
\hline Alcohol consumption: & & & $0.52^{\mathrm{b}}$ \\
\hline Yes & $28(60.87 \%)$ & 27 (67.50\%) & \\
\hline No & $18(39.13 \%)$ & $13(32.50 \%)$ & \\
\hline PSQI & $12.09 \pm 2.49$ & $11.63 \pm 2.36$ & $0.38^{\mathrm{a}}$ \\
\hline ESS & $14.33 \pm 2.46$ & $14.08 \pm 2.28$ & $0.63^{\mathrm{a}}$ \\
\hline GERD-Q & $4.09 \pm 2.98$ & $4.43 \pm 2.5 \mathrm{I}$ & $0.63^{\mathrm{a}}$ \\
\hline $\mathrm{TST} / \mathrm{min}$ & $398.33 \pm 23.03$ & $404.18 \pm 23.15$ & $0.25^{\mathrm{a}}$ \\
\hline Sleep efficiency (\%) & $73.15 \pm 5.59$ & $75.18 \pm 4.47$ & $0.07^{\mathrm{a}}$ \\
\hline \multicolumn{4}{|l|}{ Sleep structure: } \\
\hline NI (\%) & $15.04 \pm 2.09$ & $13.15 \pm 2.52$ & $0.05^{\mathrm{a}}$ \\
\hline N2 (\%) & $68.28 \pm 5.00$ & $70.53 \pm 2.14$ & $0.10^{\mathrm{a}}$ \\
\hline N3 (\%) & $8.56 \pm 2.5 \mathrm{I}$ & $10.05 \pm 2.0 \mid$ & $0.07^{\mathrm{a}}$ \\
\hline R (\%) & $7.89 \pm 3.02$ & $6.03 \pm 3.07$ & $0.07^{\mathrm{a}}$ \\
\hline $\mathrm{AHI}(/ \mathrm{h})$ & $30.24 \pm 5.45$ & $29.73 \pm 4.03$ & $0.63^{\mathrm{a}}$ \\
\hline Longest time of apnea (min) & $43.12 \pm 8.29$ & $41.26 \pm 4.60$ & $0.19^{\mathrm{a}}$ \\
\hline $\mathrm{LSaO}_{2}(\%)$ & $81.09 \pm 3.33$ & $80.08 \pm 4.35$ & $0.23^{\mathrm{a}}$ \\
\hline Demeester score & $18.23 \pm 7.77$ & $|7.7| \pm 5.15$ & $0.7 I^{\mathrm{a}}$ \\
\hline Acid reflux & $29.33 \pm 11.34$ & $27.77 \pm 13.84$ & $0.57^{\mathrm{a}}$ \\
\hline Weak acid reflux & $24.60 \pm 9.14$ & $23.89 \pm 7.95$ & $0.7 I^{a}$ \\
\hline Non-acid reflux & $2.14 \pm 1.30$ & $1.12 \pm 1.99$ & $0.09^{\mathrm{a}}$ \\
\hline VAS for cough & $55.76 \pm 10.59$ & $52.38 \pm 8.09$ & $0.10^{\mathrm{a}}$ \\
\hline Daytime cough symptom & $2.13 \pm 0.81$ & $2.03 \pm 0.80$ & $0.55^{\mathrm{a}}$ \\
\hline Nighttime cough symptom & $1.83 \pm 0.77$ & $1.73 \pm 0.82$ & $0.56^{\mathrm{a}}$ \\
\hline
\end{tabular}

Notes: ${ }^{a} t$-test; ${ }^{b}$ Chi-square test; ${ }^{*} P<0.05$ was considered statistically significant.

parameters did not differ statistically between the control and treatment groups at the baseline $(P>0.05)$.

\section{Comparisons of Sleep, GER and Cough-Related Parameters Between the Treatment Group and Control Group}

PSQI and ESS scores improved in the treatment group after 1-week treatment compared with baseline (improved by $15.14 \%$ for PSQI, Figure $2 \mathrm{~A}$; improved by $24.63 \%$ for ESS score, Figure $2 \mathrm{~B} ; P<0.05$ ), and compared with those in the control group at the same measurement time (improved by $9.20 \%$ for PSQI, Figure 2A; improved by $21.91 \%$ for ESS score, Figure 2B; $P<0.05$ ); and the improvement became stronger with the duration of therapy in the treatment group (improved by $45.16 \%$ after three months' intervention for PSQI, Figure 2A; improved by $62.39 \%$ after three months' intervention for ESS score, Figure 2B; $P<0.05)$. Compared with pre-treatment data, GERD-Q (improved by $97.80 \%$ after three months' intervention in the treatment group, $P<$ 0.05 ; improved by $48.53 \%$ after three months' intervention in the control group, $P<0.05$; Figure 3 ), VAS (improved by $81.87 \%$ after three months' intervention in the treatment group, $P<0.05$; improved by $22.91 \%$ after three months' intervention in the control group, $P<0.05$; Figure 4A), daytime cough symptom scores (improved by $89.67 \%$ after three months' intervention in the treatment group, $P<0.05$; improved by $48.28 \%$ 

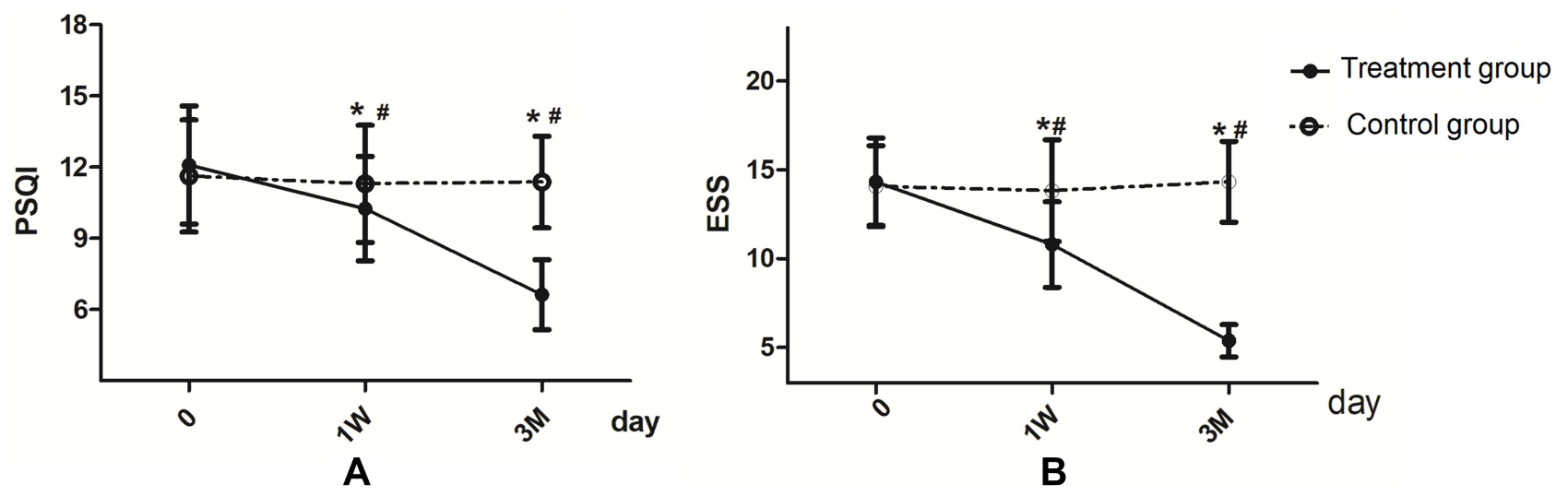

Figure 2 Comparisons of PSQI (A) and ESS score (B) between the treatment and control groups. *Indicates a significant difference between the treatment and control groups at the same measurement time $(P<0.05)$. \#Indicates a significant improvement in the treatment group when compared with the data of baseline $(P<0.05)$.

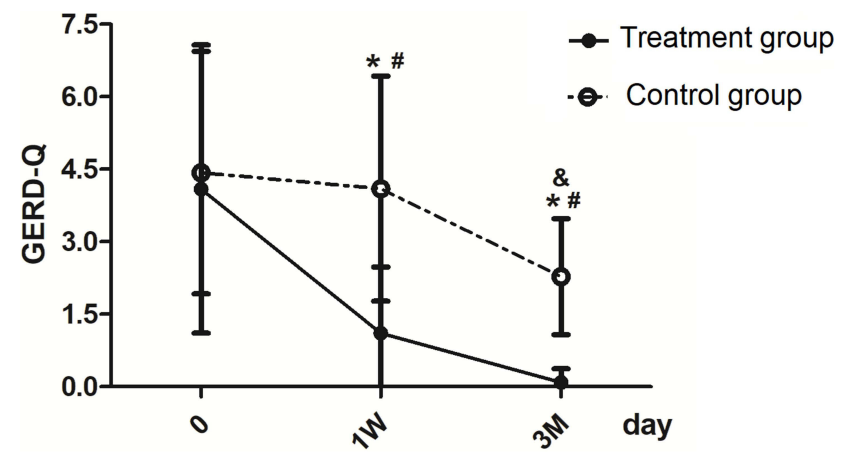

Figure 3 Comparisons of GERD-Q between the treatment and control groups. *Indicates a significant difference between the treatment and control groups at the same measurement time $(P<0.05)$. \#lndicates a significant improvement in the treatment group when compared with the data of baseline $(P<0.05)$. \&Indicates a significant improvement in the control group when compared with the data of baseline $(P<0.05)$.

after three months' intervention in the control group, $P<0.05$; Figure 4B) and night cough symptom scores (improved by $92.90 \%$ after three months' intervention in the treatment group, $P<0.05$; improved by $62.43 \%$ after three months' intervention in the control group, $P<0.05$; Figure 4C) improved after treatment in both groups $(P<0.05)$, the improvement being more significant in the treatment group $(P<0.05)$.

Significant associations between AHI and VAS scores (Figure 5A, $\mathrm{r}=0.612, \mathrm{P}<0.05$ ) and between weak acid reflux and VAS score (Figure $5 \mathrm{~B}, \mathrm{r}=0.429, \mathrm{P}<0.05$ ) were observed. Further, a significant correlation was found between weak acid reflux and AHI (Figure 5C, $\mathrm{r}=0.366, \mathrm{P}<0.05)$. VAS for cough had no significant relationship with age, BMI, PSQI, ESS, GERD-Q or the other objective sleep and reflux-related indicators (Table 2).

\section{Discussion}

The present study yielded two major findings. First, antireflux treatment and lifestyle modifications provided partial relief of chronic cough and reflux symptoms which were further resolved by CPAP in patients with OSA and concomitant GER. Second, AHI and weak acid reflux were significantly correlated with chronic cough, and AHI also showed a relationship with weak acid reflux. These findings are consistent with those of other studies showing that CPAP treatment can improve GER $^{13,33}$ and daytime sleepiness. ${ }^{13}$ Previous studies have explored the effects of CPAP on GER and OSA, but there had been no analysis focused on the effect of CPAP on chronic cough in patients with OSA and concomitant GER. The present study investigated the effects of different treatment modalities on chronic cough in patients with OSA and concomitant GER, allowing clinicians to provide CPAP therapy after determining its effect on chronic cough in these patients.

Orr et al suggested that proton pump inhibitor could improve GER symptoms, but it was not statistically significant in patients with OSA and concomitant GER. ${ }^{34}$ The CHEST Guideline and Expert Panel Report published in Chest pointed out that lifestyle modifications, weight loss, and proton pump inhibitor treatment could alleviate cough symptoms in patients with GER. ${ }^{12}$ In our study, we found that antireflux treatment and lifestyle modifications could partially relief the symptoms of cough and GER; but, it had no effect on sleep quality and daytime sleepiness in patients with OSA and concomitant GER. These results suggested that GER may be one of contributors of chronic cough, rather than the primary cause since refractory GER, such as gastrointestinal motility disorder, has been 

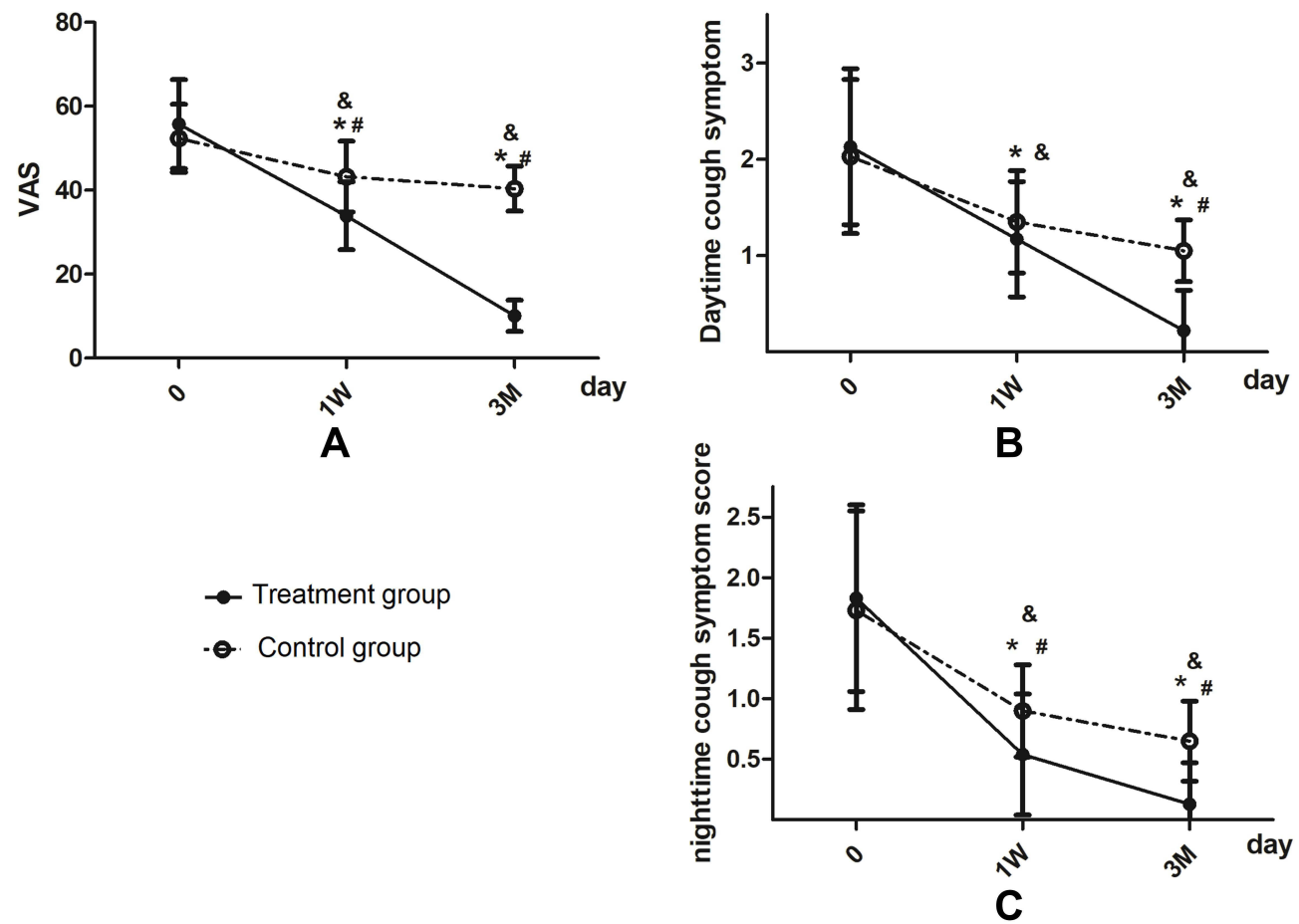

Figure 4 Comparisons of VAS for cough (A), Daytime cough symptom score (B) and Nighttime cough symptom score (C) between the treatment and control groups. *Indicates a significant difference between the treatment and control groups at the same measurement time $(P<0.05)$. \#Indicates a significant improvement in the treatment group when compared with the data of baseline $(P<0.05)$. \&Indicates a significant improvement in the control group when compared with the data of baseline $(P<0.05)$.
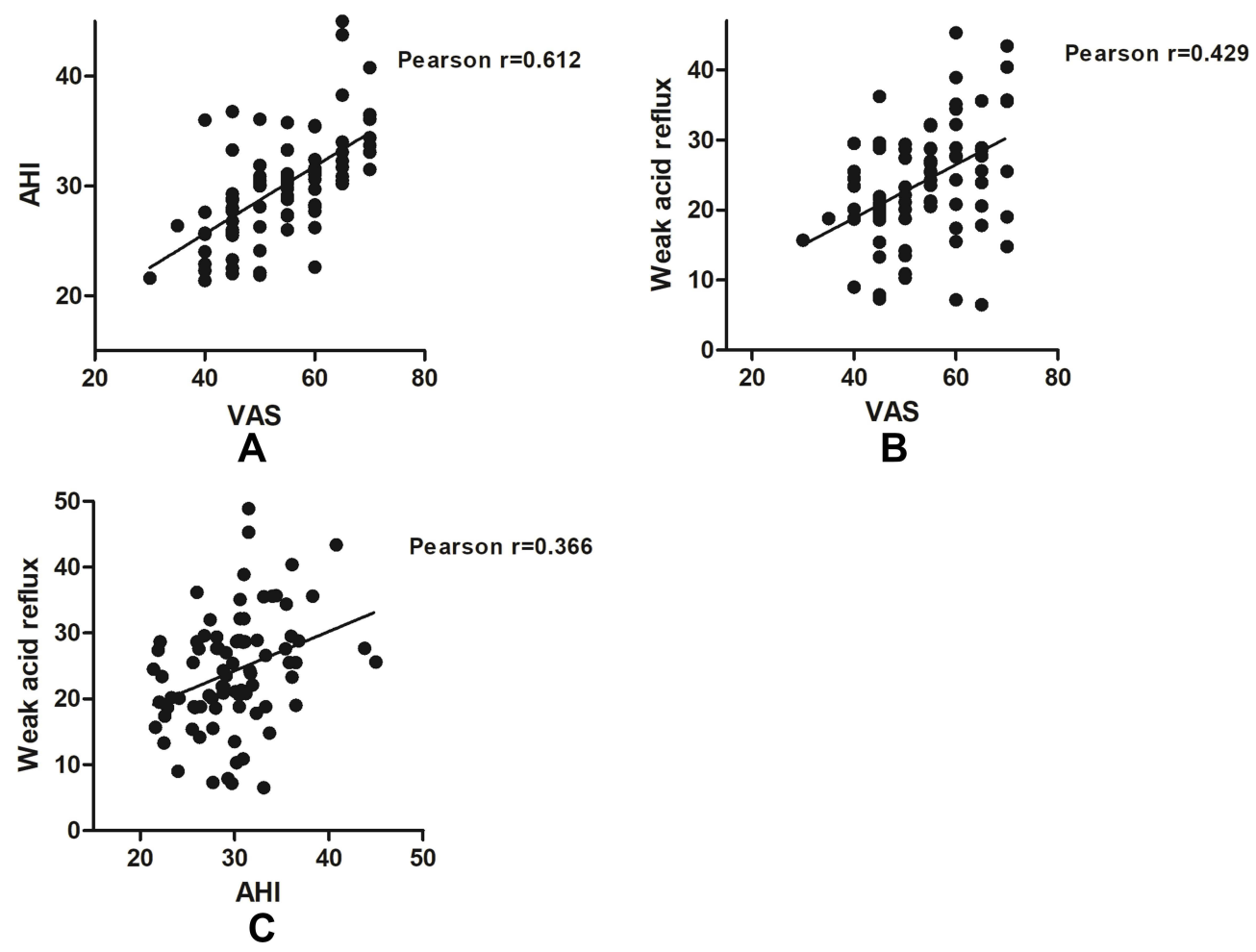

Figure 5 Correlation between AHI and VAS score $(\mathbf{A})$, weak acid reflux and VAS score (B), weak acid reflux and AHI (C). 
Table 2 The Correlation Between VAS and Other Quantitative Data

\begin{tabular}{|c|c|c|c|c|c|c|c|c|c|c|}
\hline & Age & BMI & PSQI & ESS & $\begin{array}{c}\text { GERD- } \\
\mathbf{Q}\end{array}$ & $\begin{array}{c}\text { Longest Time of } \\
\text { Apnea }\end{array}$ & LSaO & Demeester & $\begin{array}{c}\text { Acid } \\
\text { Reflux }\end{array}$ & $\begin{array}{c}\text { Non-Acid } \\
\text { Reflux }\end{array}$ \\
\hline $\mathrm{r}$ & -0.098 & 0.126 & -0.090 & 0.064 & 0.127 & 0.084 & -0.082 & -0.019 & -0.166 & -0.083 \\
$P$ & 0.369 & 0.248 & 0.409 & 0.555 & 0.244 & 0.444 & 0.455 & 0.862 & 0.127 & 0.447 \\
\hline
\end{tabular}

excluded. Tawk et al have found that GER improved after 1-week CPAP treatment in patients with OSA and concomitant GER. ${ }^{33}$ Furthermore, Tamanna et al discovered that GER and daytime sleepiness improved after 6 months of CPAP treatment in patients with OSA and concomitant GER. ${ }^{13}$ We assessed the association among chronic cough, GER, and treatment response in patients with OSA and concomitant GER, and found that sleep parameters (such as sleep quality and daytime sleepiness), symptoms of chronic cough and GER were all markedly improved after 3-month CPAP treatment in present study. A meta-analysis has demonstrated that GER is involved in the pathogenesis of OSA in patients with OSA and concomitant GER. ${ }^{35}$ Radovanovic et al believed that although OSA and GER might not be causally related, the two disorders probably interact. ${ }^{36}$ Our data suggested that antireflux treatment and lifestyle modifications provided partial relief of cough and GER symptoms which were further resolved by CPAP. We speculated that OSA might be the primary cause of chronic cough, and GER might only play a role of contributor in patients with OSA and concomitant GER.

We discovered the associations between AHI and VAS, weak acid reflux and VAS, and AHI and weak acid reflux, and found there was no significant correlation between cough and age, BMI, PSQI, ESS, GERD-Q and the other objective sleep and reflux-related indicators in this study. These findings are consistent with those of several previous studies, but not entirely. ${ }^{10,37,38}$ Previous studies have reported the association between AHI and GER using symptoms, ${ }^{4}$ responses to anti-GER medication, ${ }^{39}$ 24-h esophageal $\mathrm{pH}$ monitoring, ${ }^{39}$ or endoscopic findings ${ }^{10}$ to define GER, rather than 24-h MII-pH as in the present study. The 24-h MII-pH monitoring can accurately distinguish and analyze several forms of reflux (such as acid reflux, weak acid reflux, and non-acid reflux). ${ }^{40}$ The wide use of 24-h MII-pH monitoring has shown that not all forms of reflux can cause symptoms in patients with GER, and the 24-h esophageal $\mathrm{pH}$ monitoring may be far from perfect for the diagnosis of patients with extraesophageal symptoms. Compared to patients with GER without cough, the frequency of weak acid reflux was significantly higher in patients with cough. ${ }^{41}$ This probably reveals why some reflux episodes in patients with GER cause cough while others do not. Our findings suggested that both AHI and weak acid reflux play important roles in the development of chronic cough in patients with OSA and concomitant GER.

Several studies have suggested that OSA causes GER, rather than the reverse, while there are no objective parameters to assess AHI and reflux episodes in these studies. $^{35,42,43}$ The mechanism by which CPAP relieve the symptom of chronic cough in patients with OSA and concomitant GER is not fully understood. Chronic cough is multifactorial and etiologies includes pharyngeal and laryngeal hypersensitivity. This could occur due to a variety of irritants including acid. Several studies have demonstrated that CPAP can make the lower esophageal sphincter (LES) less prone to reflux via increasing barrier pressure to reflux and reducing LES relaxation time. ${ }^{44,45}$ We speculated that CPAP determines chronic cough resolution probably by improving upper airway collapse, and reducing the effect of intrathoracic and abdominal pressure, and further promoting the barrier function of LES, and then alleviating weak acid reflux in patients with OSA.

Additionally, there are still several issues to be addressed. First, patient population was young with mean age about $36-37$ years in present study. This is not typical of most moderate-severe OSA study populations. What was the reason for this? This may be related to the interaction between OSA and GER. Shepherd et al found that the severity of GER symptom was correlated with the severity of $\mathrm{AHI},{ }^{46}$ that is, the higher the AHI index, the more severe the reflux symptom. Further fundamental study is necessary to elucidate this mechanism in the future. Second, the baseline of GERD-Q in this study was less than 8 in both groups, while the GERD-Q should be more than 8 to be GER disease. The possible reasons for this are as follows: (1) the GER symptoms were divided into two parts, esophageal and extra-esophageal symptoms. GERD-Q consists of six questions: heartburn, reflux, epigastric pain, nausea, sleep disorder, and anti- 
reflux treatment use within the past 1 week; $5 / 6$ of the questions were related to esophageal symptoms. It indicates that GERD-Q tends to diagnose patients with typical esophageal symptoms more than those with atypical esophageal symptoms. And cough is one of the extraesophageal symptoms of GER. (2)24-h MII-pH monitoring further discovered that the significant abnormalities were weak acid reflux in OSA patients with GER in present study. GER esophageal symptoms are atypical in patients with OSA and concomitant GER, which might explain why both groups' baseline GERD-Q was less than 8. Further, it also indicated that the GERD-Q score alone could not diagnose GER in patients with OSA and concomitant GER. Patients with probable OSA and GER should have their MII-pH level monitored for 24 hours. Third, previous studies have found a sex difference in the incidence of OSA. ${ }^{4,48}$ However, sex did not affect the therapeutic effect of cough in patients with OSA and concomitant GER in present study. It suggested that sex could affect the incidence of OSA, but CPAP therapy was equally important for cough in male and female patients with OSA and concomitant GER.

This study had several limitations. First, this was a singlecenter retrospective study. Further fundamental studies and large-scale prospective studies are necessary to reach a more definitive conclusion in the future. Second, there was no blank control group. However, it would be unethical to withhold therapy from patients. Third, the subjective parameters, such as sleep quality, daytime sleepiness, GER-related questionnaire, and cough-related questionnaires, could be influenced by patients' judgment. However, these questionnaires are simple and practical to perform, and there is no objective cough-related monitoring up till now. Moreover, professional technicians use these questionnaires strictly and accurately according to the instructions in our study.

\section{Conclusion}

In summary, our results indicate that CPAP improved the symptoms of chronic cough and GER in patients with OSA and concomitant GER. Meanwhile, AHI and weak acid reflux may be important factors affecting the therapeutic effect of chronic cough in patients with OSA and concomitant GER. The probable mechanism of cough resolution with CPAP is the improvement of upper airway collapse, and reducing the effect of intrathoracic and abdominal pressure, further promoting the barrier function of LES and then alleviating weak acid reflux in patients with OSA. Further fundamental studies and large-scale prospective studies are necessary to reach a more definitive conclusion in the future.

\section{Abbreviations}

24-h MII-pH, 24-h esophageal multichannel intraluminal impedance-pH; AHI, apnea-hypopnea index; CPAP, continuous positive airway pressure; ESS, Epworth Sleepiness Scale; GER, gastroesophageal reflux; GERD-Q, Gastroesophageal reflux disease questionnaire; LES, Lower esophageal sphincter; OSA, obstructive sleep apnea; PSQI, Pittsburgh Sleep Quality Index; VAS, Visual analog scale.

\section{Ethics Approval and Informed Consent}

Informed consent was waived owing to the retrospective nature of the study. The patient data accessed was maintained with confidentiality. This study was conducted in accordance with the World Medical Association Declaration of Helsinki and approved by the grass-roots ethics committee of the First Affiliated Hospital of Zhengzhou University.

\section{Acknowledgments}

We thank Jiao Su's classmates, the statisticians (Xin Chen, Dalian Medical University, Dalian, China; Bing Huang, Dalian Runsheng Kangtai Medical Lab Co. Ltd., Dalian, China), for their help in the statistical analysis.

\section{Author Contributions}

All authors made a significant contribution to the work reported, whether that is in the conception, study design, execution, acquisition of data, analysis and interpretation, or in all these areas; took part in drafting, revising or critically reviewing the article; gave final approval of the version to be published; have agreed on the journal to which the article has been submitted; and agree to be accountable for all aspects of the work.

\section{Funding}

Supported by grants No. 81800091 and U1804195 from National Natural Science Foundation of China.

\section{Disclosure}

All authors declared that the research was conducted in the absence of any commercial or financial relationships that 
could be construed as a potential conflict of interest and reported no conflicts of interest for this work.

\section{References}

1. Chung KF, Pavord ID. Chronic cough prevalence, pathogenesis, and causes of chronic cough. Lancet. 2008;371(9621):1364-1374. doi:10.1016/S0140-6736(08)60595-4

2. Song WJ, Chang YS, Faruqi S, et al. The global epidemiology of chronic cough in adults: a systematic review and meta-analysis. Eur Respir J. 2015;45(5):1479-1481. doi:10.1183/09031936.00218714

3. Michaudet C, Malaty J. Chronic cough: evaluation and management. Am Fam Physician. 2017;96(9):575-580.

4. Gouveia CJ, Yalamanchili A, Ghadersohi S, et al. Are chronic cough and laryngopharyngeal reflux more common in obstructive sleep apnea patients? Laryngoscope. 2019;129(5):1244-1249. doi:10.1002/lary.27557

5. Javaheri S, Barbe F, Campos-Rodriguez F, et al. Sleep apnea: types, mechanisms, and clinical cardiovascular consequences. J Am Coll Cardiol. 2017;69(7):841-858. doi:10.1016/j.jacc.2016.11.069

6. Dong R, Dong Z, Liu H, et al. Prevalence, risk factors, outcomes, and treatment of obstructive sleep apnea in patients with cerebrovascular disease: a systematic review. J Stroke Cerebrovasc Dis. 2018;27 (6):1471-1480. doi:10.1016/j.jstrokecerebrovasdis.2017.12.048

7. Chan KK, Ing AJ, Laks L, et al. Chronic cough in patients with sleep-disordered breathing. Eur Respir J. 2010;35(2):368-372. doi:10.1183/09031936.00110409

8. Shi C, Liang S, Xu X, et al. Cough hypersensitivity in patients with obstructive sleep apnea hypopnea syndrome. Sleep Breath. 2019;23 (1):33-39. doi:10.1007/s11325-018-1641-7

9. Sabaté JM, Jouët P, Merrouche M, et al. Gastroesophageal reflux in patients with morbid obesity: a role of obstructive sleep apnea syndrome? Obes Surg. 2008;18(11):1479-1484. doi:10.1007/ s11695-008-9508-9

10. Kim Y, Lee YJ, Park JS, et al. Associations between obstructive sleep apnea severity and endoscopically proven gastroesophageal reflux disease. Sleep Breath. 2018;22(1):85-90. doi:10.1007/s11325-0171533-2

11. Gilani S, Quan SF, Pynnonen MA, et al. Obstructive sleep apnea and gastroesophageal reflux: a multivariate population-level analysis. Otolaryngol Head Neck Surg. 2016;154(2):390-395. doi:10.1177/ 0194599815621557

12. Kahrilas P, Altman KW, Chang AB. Chronic cough due to gastroesophageal reflux in adults: CHEST guideline and expert panel report. Chest. 2016;150(6):1341-1360. doi:10.1016/j. chest.2016.08.1458

13. Tamanna S, Campbell D, Warren R, et al. Effect of CPAP therapy on symptoms of nocturnal gastroesophageal reflux among patients with obstructive sleep apnea. J Clin Sleep Med. 2016;12(9):1257-1261. doi: $10.5664 /$ jcsm. 6126

14. Wang L, Han H, Wang G, et al. Relationship between reflux diseases and obstructive sleep apnea together with continuous positive airway pressure treatment efficiency analysis. Sleep Med. 2020;75:151-155. doi:10.1016/j.sleep.2020.07.024

15. Sundar KM, Willis AM, Smith S, et al. A Randomized, Controlled, Pilot Study of CPAP for patients with chronic cough and obstructive sleep apnea. Lung. 2020;198(3):449-457. doi:10.1007/s00408-02000354-1

16. The asthma group of Chinese Thoracic Society. Guideline for the diagnosis and treatment of cough (2015). Chin J Tuberc Respir Dis. 2016;39(5):323-354.

17. World Health Organisation. Guidelines for Controlling and Monitoring the Tobacco Epidemic. Geneva: Tobacco or Heahh Programme, WHO; 1997.
18. World Health Organisation. ICD-10 Classification of Mental and Behavioural Disorders: Clinical Descriptions and Diagnostic Guidelines. Geneva, Switzerland: World Health Organization; 1992.

19. Mollayeva T, Thurairajah P, Burton K, et al. The Pittsburgh sleep quality index as a screening tool for sleep dysfunction in clinical and non-clinical samples: a systematic review and meta-analysis. Sleep Med Rev. 2016;25:52-73. doi:10.1016/j.smrv.2015.01.009

20. Johns MW. A new method for measuring daytime sleepiness: the Epworth sleepiness scale. Sleep. 1991;14(6):540-545. doi:10.1093/ sleep/14.6.540

21. Teklu M, Gouveia CJ, Yalamanchili A, et al. Predicting obstructive sleep apnea status with the reflux symptom index in a sleep study population. Laryngoscope. 2020;130(12):E952-E957. doi:10.1002/ lary. 28592

22. Neto R, Herbella F, Schlottmann F, et al. Does DeMeester score still define GERD? Dis Esophagus. 2019;32(5):doy118. doi:10.1093/dote/ doy 118

23. Gyawali CP, Kahrilas PJ, Savarino E, et al. Modern diagnosis of GERD: the Lyon consensus. Gut. 2018;67(7):1351-1362. doi:10.1136/gutjnl-2017-314722

24. Sifrim D, Castell D, Dent J, Kahrilas PJ. Gastro-oesophageal reflux monitoring: review and consensus report on detection and definitions of acid, non-acid, and gas reflux. Gut. 2004;53(7):1024-1031. doi:10.1136/gut.2003.033290

25. Berry RB, Brooks R, Gamaldo CE, et al; for the American Academy of Sleep Medicine. The AASM Manual for the Scoring of Sleep and Associated Events: Rules, Terminology, and Technical Specifications. Version 2.4. Darien, IL: American Academy of Sleep Medicine; 2017.

26. Chawla S, Tessarolo Silva F, Amaral Medeiros S, Mekary RA, Radenkovic D. The effect of low-fat and low-carbohydrate diets on weight loss and lipid levels: a systematic review and meta-analysis. Nutrients. 2020;12(12):3774. doi:10.3390/nu12123774

27. Clifton PM, Condo D, Keogh JB. Long term weight maintenance after advice to consume low carbohydrate, higher protein diets-a systematic review and meta analysis. Nutr Metab Cardiovasc Dis. 2014;24(3):224-235. doi:10.1016/j.numecd.2013.11.006

28. Kline CE, Crowley EP, Ewing GB, et al. The effect of exercise training on obstructive sleep apnea and sleep quality: a randomized controlled trial. Sleep. 2011;34(12):1631-1640. doi:10.5665/ sleep. 1422

29. Yang H, Liu Y, Zheng H, Liu G, Mei A. Effects of 12 weeks of regular aerobic exercises on autonomic nervous system in obstructive sleep apnea syndrome patients. Sleep Breath. 2018;22(4):1189-1195. doi:10.1007/s11325-018-1736-1

30. Neumannova K, Hobzova M, Sova M, Prasko J. Pulmonary rehabilitation and oropharyngeal exercises as an adjunct therapy in obstructive sleep apnea: a randomized controlled trial. Sleep Med. 2018;52:92-97. doi:10.1016/j.sleep.2018.03.022

31. Sawyer AM, Gooneratne NS, Marcus CL, Ofer D, Richards KC, Weaver TE. A systematic review of CPAP adherence across age groups: clinical and empiric insights for developing CPAP adherence interventions. Sleep Med Rev. 2011;15(6):343-356. doi:10.1016/j. smrv.2011.01.003

32. Bakker JP, Weaver TE, Parthasarathy S, Aloia MS. Adherence to CPAP: what should we be aiming for, and how can we get there? Chest. 2019;155(6):1272-1287. doi:10.1016/j.chest.2019.01.012

33. Tawk M, Goodrich S, Kinasewitz G, et al. The effect of 1 week of continuous positive airway pressure treatment in obstructive sleep apnea patients with concomitant gastroesophageal reflux. Chest. 2006;130(4):1003-1008. doi:10.1378/chest.130.4.1003

34. Orr WC, Robert JJT, Houck JR, et al. The effect of acid suppression on upper airway anatomy and obstruction in patients with sleep apnea and gastroesophageal reflux disease. J Clin Sleep Med. 2009;5 (4):330-334. doi:10.5664/jcsm.27543 
35. Wu ZH, Yang XP, Niu X, et al. The relationship between obstructive sleep apnea hypopnea syndrome and gastroesophageal reflux disease: a meta-analysis. Sleep Breath. 2019;23(2):389-397. doi:10.1007/ s11325-018-1691-x

36. Radovanovic D, Pini S, Rizzi M, et al. Prevalence and influence of gastroesophageal reflux symptoms on sleep apnea and sleepiness. Eur J Intern Med. 2021;84:115-117. doi:10.1016/j.ejim.2020.11.022

37. Vela MF, Kramer JR, Richardson PA, et al. Poor sleep quality and obstructive sleep apnea in patients with GERD and barrett's esophagus. Neurogastroenterol Motil. 2014;26(3):346-352. doi:10.1111/ nmo. 12265

38. Ju G, Yoon I, Lee SD, et al. Relationships between sleep disturbances and gastroesophageal reflux disease in Asian sleep clinic referrals. J Psychosom Res. 2013;75(6):551-555. doi:10.1016/j. jpsychores.2013.10.004

39. Faruqi S, Fahim A, Morice AH. Chronic cough and obstructive sleep apnoea: reflux-associated cough hypersensitivity?. Eur Respir J. 2012;40(4):1049-1050. doi:10.1183/09031936.00025012

40. Boeckxstaens GE, Smout A. Systematic review: role of acid, weakly acidic and weakly alkaline reflux in gastro-oesophageal reflux disease. Aliment Pharmacol Ther. 2010;32:332-343. doi:10.1111/ j.1365-2036.2010.04358.x

41. Yu Q, Liu Z, Liao J, et al. The characteristics of high-resolution esophageal manometry and multichannel intraluminal esophageal impedance and $\mathrm{pH}$ monitoring in gastroesophageal reflux cough. Chin J Gastroenterol Hepatol. 2015;24(10):1196-1999.
42. Xavier SD, Moraes JP, Eckley CA. Prevalence of signs and symptoms of laryngopharyngeal reflux in snorers with suspected obstructive sleep apnea. Braz J Otorhinolaryngol. 2013;79:589-593. doi:10.5935/1808-8694.20130105

43. Laohasiriwong S, Johnston N, Woodson BT. Extra-esophageal reflux, nose score, and sleep quality in an adult clinic population. Laryngoscope. 2013;123(12):3233-3238. doi:10.1002/lary.24236

44. Shepherd KL, Holloway RH, Hillman DR, et al. The impact of continuous positive airway pressure on the lower esophageal sphincter. Am J Physiol Gastrointest Liver Physiol. 2007;292(5): G1200-G1205. doi:10.1152/ajpgi.00476.2006

45. Shepherd K, Hillman D, Holloway R, et al. Mechanisms of nocturnal gastroesophageal reflux events in obstructive sleep apnea. Sleep Breath. 2011;15(3):561-570. doi:10.1007/s11325-010-0404-x

46. Shepherd KL, James AL, Musk AW, Hunter ML, Hillman DR, Eastwood PR. Gastro-oesophageal reflux symptoms are related to the presence and severity of obstructive sleep apnoea. J Sleep Res. 2011;20(1pt2):241-249. doi:10.1111/j.1365-2869.2010.00843.x

47. Fietze I, Laharnar N, Obst A, et al. Prevalence and association analysis of obstructive sleep apnea with gender and age differences - results of SHIP-trend. J Sleep Res. 2019;28(5):e12770. doi:10.1111/ jsr. 12770

48. Wächter M, Kantelhardt JW, Bonsignore MR, et al. Unique sleep-stage transitions determined by obstructive sleep apnea severity, age and gender. J Sleep Res. 2020;29(2):e12895. doi:10.1111/ jsr. 12895

\section{Publish your work in this journal}

Nature and Science of Sleep is an international, peer-reviewed, open access journal covering all aspects of sleep science and sleep medicine, including the neurophysiology and functions of sleep, the genetics of sleep, sleep and society, biological rhythms, dreaming, sleep disorders and therapy, and strategies to optimize healthy sleep.
The manuscript management system is completely online and includes a very quick and fair peer-review system, which is all easy to use. Visit http://www.dovepress.com/testimonials.php to read real quotes from published authors. 